\title{
Actinomicosis pélvica invasiva. Un desafío para el ginecólogo
}

\section{Invasive pelvic actinomycosis. A challenge for the gynecologist}

\author{
Patricio Cisterna ${ }^{1}$ y Alfredo Ovalle $2 *$ \\ ${ }^{1}$ Servicio de Obstetricia y Ginecología, Hospital San Juan de Dios; ${ }^{2}$ Departamento de Obstetricia y Ginecología Centro, Facultad de Medicina, \\ Universidad de Chile. Santiago, Chile
}

\section{Resumen}

La actinomicosis pélvica es una infección bacteriana supurativa crónica, producida por especies de Actinomyces, principalmente Actinomyces israelii, que afecta el aparato genital interno y las estructuras vecinas, asociada al uso prolongado de dispositivo intrauterino sin control en casi la totalidad de los casos descritos en mujeres. La actinomicosis pélvica suele presentarse como un absceso tubo-ovárico y con menor frecuencia como una actinomicosis pélvica invasiva (API). La API se propaga por contigüidad desde el aparato genital hacia las vísceras adyacentes, originando un tumor pélvico difuso, de consistencia leñosa, pseudotumoral, que a menudo se confunde con una neoplasia pélvica. La API representa un gran desafío para el ginecólogo por las dificultades en su diagnóstico y manejo. Se presentan dos casos de API y se revisan los procedimientos diagnósticos y terapéuticos recomendados actualmente para el enfrentamiento de esta patología.

Palabras clave: Actinomicosis pélvica invasiva. Dispositivo intrauterino. Diagnóstico diferencial.

\section{Abstract}

Pelvic actinomycosis (PA) is a chronic suppurative bacterial infection, produced by Actinomyces, mainly Actinomyces israelii. It affects the internal genital tract, adjacent structures and is associated with a prolonged intrauterine device use with an inadequate control in almost all described cases in women. Pelvic actinomycosis usually presents as a tube ovarian abscess and less frequently as invasive pelvic actinomycosis (IPA). The IPA spreads contiguously from the genital tract to adjacent viscera, causing a diffuse, woody, pseudotumoral pelvic tumor that is frequently confused with a pelvic neoplasm. The IPA represents a great challenge for the gynecologist due to the difficulties in the diagnosis and management of this disease. Two cases of IPA are presented and the currently recommended diagnostic and therapeutic procedures for dealing with this pathology are reviewed.

Key words: Invasive pelvic actinomycosis. Intrauterine device. Differential diagnosis.

\section{Introducción}

La actinomicosis es una infección bacteriana supurativa crónica, producida por especies de Actinomyces, principalmente Actinomyces israelii, bacilo filamentoso, grampositivo anaerobio, no esporulado, que coloniza la orofaringe, el tracto gastrointestinal y el canal vaginal $^{1-9}$. La actinomicosis ocurre con mayor frecuencia en las regiones cérvico-facial, torácica, abdominal y pélvica'. Generalmente se manifiesta con formación de granulomas abscesificados de crecimiento lento, fístulas y fibrosis densa en la modalidad invasiva 1 . Los
E-mail: alfredoovalle @gmail.com
Disponible en internet: 02-08-2021 Rev Chil Obstet Ginecol. 2021;86(3):322-325

www. rechog.com 
factores predisponentes son apendicitis perforada, diverticulitis, cuerpo extraño (como dispositivo intrauterino [DIU], pesario, inserto de espiral usado en esterilización histeroscópica), cirugía previa y neoplasia $3-8,10-18$.

La actinomicosis pélvica ocurrió en el $17 \%$ de las pacientes con absceso tubo-ovárico en el Hospital Clínico San Borja Arriarán ${ }^{4}$ y se presenta asociada al uso prolongado de DIU sin control en casi la totalidad de los casos descritos en mujeres ${ }^{3-8,10-18}$, al actuar como cuerpo extraño. En esta serie la modalidad actinomicosis pélvica invasiva (API) con fibrosis densa fue aislada ${ }^{4}$. En la API se propaga la infección desde el aparato genital hasta los órganos vecinos, por diseminación contigua mediante granulomas que contienen múltiples colonias de Actinomyces ${ }^{1,4}$. Se origina una masa pélvica fibrosa, descrita como «pelvis congelada o sellada", por la incapacidad de liberar las estructuras comprometidas durante la cirugía. La API se denominada «pseudotumoral» porque se presenta como tumor pélvico complejo con características imagenológicas inespecíficas, que a menudo se confunden con neoplasia ${ }^{2-4,6,7,11-22}$ y supuestas metástasis, que corresponden a la diseminación hematógena a distancia de la infección, por lo que el diagnóstico y el manejo representan un gran desafío para el ginecólogo.

El diagnóstico se hace por el hallazgo de gránulos en el pus o en secciones histológicas de la muestra quirúrgica ${ }^{1,3-5,12,17,18,23}$. Los filamentos de las bacterias se tiñen con técnicas especiales, demostrando que los gránulos están compuestos por bacterias en ramificación ${ }^{3-5,13-15}$. En ausencia de gránulos, el hallazgo de bastones y filamentos ramificados grampositivos en el frotis es sugerente de Actinomyces ${ }^{1,13}$.

El tratamiento es con antibióticos betalactámicos de uso prolongado $1,3,4,7,10,11,13,17,22$, comúnmente administrados por vía intravenosa los primeros días, para luego continuar con la vía oral hasta completar 3 a 6 meses. La penicilina $G$ y sus derivados son los antimicrobianos de elección ${ }^{3,7,8,10,11,13,21}$. La API responde bien a esta terapia y habitualmente desaparecen por completo los tumores actinomicóticos.

Presentamos dos casos de API atendidos en el servicio de obstetricia y ginecología del Hospital San Juan de Dios, en los que fue necesario hacer diagnóstico diferencial con cáncer. Se revisan los métodos diagnósticos y el manejo de estos complicados y difíciles casos de origen ginecológico.

\section{Caso clínico 1}

Mujer de 43 años, multípara, dos partos vaginales, usuaria de DIU (tipo TCu) por 14 años sin recambio ni control. Consultó por dolor en el hemiabdomen bajo, baja de peso, afebril, flujo genital de mal olor y sin evacuación intestinal durante 7 días. Examen manual pélvico: DIU in situ, útero y anexo izquierdo aumentados de tamaño, indurados y fijos. La tomografía computarizada (TC) informó de engrosamiento en recto superior de aspecto neoplásico, quiste anexial izquierdo, sin signos de obstrucción intestinal. Los exámenes de laboratorio reportaron: hematocrito/hemoglobina $32 \% / 10,5 \mathrm{~g} / \mathrm{dl}$, glóbulos blancos $16.300 / \mathrm{mm}^{3}$, velocidad horaria de sedimentación $65 \mathrm{~mm} / \mathrm{h}$, proteína C reactiva $78 \mathrm{mg} / \mathrm{l}$ (rango normal: 0-10 mg/dl), creatinina $0,48 \mathrm{mg} / \mathrm{dl}$, antígeno carcinoembrionario $<0,5 \mathrm{ng} / \mathrm{ml}$ (normal: < $10 \mathrm{ng} / \mathrm{ml}$ ) y CA-125 $28 \mathrm{U} / \mathrm{ml}$. La colonoscopia se informó como sospecha de infiltración en recto medio por lesión extrínseca en cara anterior. Una biopsia descartó lesión neoplásica. La tomografía por emisión de positrones no reportó alteraciones del metabolismo. En la evaluación por la unidad de ginecología oncología se planteó el diagnóstico de API. Se realizó laparoscopia diagnóstica y se encontró pelvis congelada. La biopsia se informó como epiplón con infiltrado inflamatorio crónico con focos supurados y presencia de colonias bacterianas de tipo Actinomyces, sin evidencia de neoplasia maligna. Se instauró tratamiento médico con ceftriaxona $2 \mathrm{~g} /$ día por vía intravenosa, metronidazol $500 \mathrm{mg} / 8 \mathrm{~h}$ por vía intravenosa por 14 días, y luego amoxicilina-ácido clavulánico $875 / 125$ mg/12 h por vía oral por 3 meses. La TC de control posterior al tratamiento reveló una imagen nodular heterogénea en la región lateral izquierda del útero, de 4,5 ×4,3 cm. Al año de seguimiento, la resolución de las lesiones es completa.

\section{Caso clínico 2}

Mujer de 58 años, multípara, tres partos eutócicos, menopausia a los 53 años. Usuaria de DIU (tipo TCu) por 12 años, retirado a los 54 años. Presentó dolor pélvico de predominio en la fosa iliaca derecha, y baja de peso de 10 kilos en 4 meses. El examen ginecológico mostró cérvix inmóvil, útero y anexo derecho que constituían una masa difusa, de consistencia pétrea, y parametrio derecho acortado. Los exámenes de laboratorio reportaron: hematocrito/hemoglobina $34 \% / 10,2 \mathrm{~g} / \mathrm{dl}$, velocidad horaria de sedimentación $104 \mathrm{~mm} / \mathrm{h}$, proteína $C$ reactiva $160 \mathrm{mg} / \mathrm{l}$, creatinina 
0,99 mg/dl y CA-125 $26 \mathrm{U} / \mathrm{ml}$. La colonoscopia evidenció compresión extrínseca de sigmoides distal con obstrucción infranqueable. La TC abdomen y pelvis informó de masa sólida heterogénea en la región anexial derecha, de $6 \mathrm{~cm}$, con aumento de la densidad de la grasa circundante, sin plano de clivaje, con vejiga sugerente de neoplasia e hidroureteronefrosis moderada a la derecha. La TC de tórax informó de múltiples nódulos pulmonares bilaterales, algunos con cavitaciones, de hasta $16 \mathrm{~mm}$, compatibles con localizaciones secundarias, sin derrame ni engrosamiento pleural. Se planteó un probable tumor ovárico, observación de cáncer metastásico o API. Se hizo laparotomía diagnóstica, en la que se encontró pelvis sellada y se tomaron biopsias de nódulos en el peritoneo parietal y el epiplón. La biopsia informó de hallazgos sugerentes de actinomicosis, sin evidencia de neoplasia maligna. Se instauró tratamiento con ceftriaxona $2 \mathrm{~g} /$ día por vía intravenosa, metronidazol $500 \mathrm{mg} / 8 \mathrm{~h}$ por vía intravenosa por 14 días y luego amoxicilina-ácido clavulánico 875/125 mg/12 h por vía oral por 3 meses. Los controles seriados demostraron buena respuesta, regresando las lesiones nodulares pulmonares, el plastrón inflamatorio y la masa anexial.

\section{Discusión}

Los signos y síntomas de la API son inespecíficos; habitualmente son de larga evolución y las manifestaciones dependen del órgano comprometido por esta infección crónica.

Los dos casos aquí descritos presentaron una tumoración abdomino-pélvica de consistencia pétrea, que es la modalidad más frecuente descrita en la literatura, en ocasiones asociada con plastrón pélvico $4,6,7,13,16,17,19-22$.

En el caso 1, la paciente no tuvo evacuación intestinal durante 7 días. Esta alteración del tránsito intestinal, también descrita por otros autores, ocurre cuando por compresión extrínseca del tumor se compromete el recto sigmoides ${ }^{13}$. Si el tumor es de mayor tamaño puede causar estenosis rectosigmoidea segmentaria y obstrucción intestinal baja, que suele confundirse con cáncer rectal 2,6,13,16,17.

En el caso 2, la lesión actinomicótica comprometió un anexo y, por la presencia de múltiples nódulos pulmonares bilaterales en la TC, se sospechó un cáncer de ovario con metástasis pulmonar. Sin embargo, estos nódulos correspondieron a la diseminación hematógena de la actinomicosis y remitieron totalmente luego del tratamiento antibiótico realizado, tal como se demostró por la desaparición de estas imágenes en la TC de control. Este caso es uno de los pocos reportes publicados en que se da a conocer la diseminación sistémica de la API, con localizaciones secundarias, como el hígado y el pulmón ${ }^{12,21,22}$.

El diagnóstico de API se sospecha en toda paciente usuaria de DIU por un tiempo superior a 10 años, habitualmente sin control $^{3-8,10-18}$, con tumor ginecológico, que en la exploración pélvica manual es de contornos imprecisos, aplastronado, de consistencia firme leñosa (casos 1 y 2) $3,4,6,7,16,24$. Se confirma el diagnóstico por biopsia quirúrgica de los granulomas, obtenida por laparoscopía exploradora (caso 1), laparotomía explo-

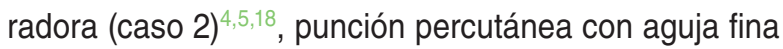
o guiada por ultrasonido ${ }^{3,22}$, o por biopsia de endometrio tomada por vía vaginal con Pipelle ${ }^{22,23}$, procedimiento ambulatorio, fácil de realizar y de alta sensibilidad. Las imágenes obtenidas por ecografía, y especialmente por TC, son complementarias para hacer el diagnóstico diferencial entre API y cáncer de órganos pélvicos, así como para controlar la evolución y la respuesta al tratamiento ${ }^{3,17,24-27}$. En los exámenes de laboratorio, las pacientes con API presentan con frecuencia anemia, leucocitosis y elevación de la velocidad horaria de sedimentación y de la proteína $C$ reactiva (casos 1 y 2), $7,1,17$.

La desaparición total de los tumores con el tratamiento antibiótico ${ }^{28,29}$ confirma que los tumores pélvi$\cos$ (casos 1 y 2) y los nódulos pulmonares (caso 2) son por actinomicosis.

Por lo anterior, no se recomienda la laparotomía quirúrgica debido al alto riesgo de lesiones iatrogénicas, intestinales y de vías urinarias, como consecuencia de la adherencia íntima de las vísceras comprometidas ${ }^{2,4}$. La laparoscopía exploradora (caso 1) y la laparotomía exploradora (caso 2) se realizan en caso de dudas diagnósticas, para descartar una neoplasia maligna y tomar muestras para biopsia. La indicación quirúrgica al término del tratamiento antibiótico programado solo se recomienda en aislados casos acotados de infertilidad, u otros, y alejada del episodio de API.

En conclusión, la API o actinomicosis pseudotumoral es una infrecuente presentación de la actinomicosis pélvica que se confunde con cáncer de órganos pélvicos. Su diagnóstico y su tratamiento representan un desafío para el ginecólogo. Se sospecha en usuarias de DIU por tiempo prolongado que presentan un tumor pélvico abdominal de consistencia leñosa. El diagnóstico se confirma mediante biopsia de muestras obtenidas por laparotomía o laparoscopía exploradoras, punción percutánea o biopsia de endometrio. El compromiso de órganos pélvicos y la extensión de la lesión se establecen con pruebas de imagen, especialmente 
con TC. El tratamiento es con antibióticos de uso prolongado, preferentemente penicilina $\mathrm{G}$ y sus derivados, que logran la remisión total de los tumores actinomicóticos pélvicos y de los eventuales tumores a distancia. La cirugía ampliada no se recomienda, dada la eficacia del tratamiento antibiótico y por la alta frecuencia de lesiones iatrogénicas que se producen en el intento, facilitadas por la adherencia íntima de los órganos comprometidos y la inexistencia de tejidos sanos que permitan su separación. El diagnóstico acertado y oportuno permite el tratamiento eficaz con antibióticos, evita la cirugía y las complicaciones asociadas, y mejora el pronóstico de esta enfermedad.

\section{Financiación}

Los autores declaran que no recibieron financiación para este trabajo.

\section{Conflicto de intereses}

Los autores declaran que no existen conflictos de intereses.

\section{Responsabilidades éticas}

Protección de personas y animales. Los autores declaran que para esta investigación no se han realizado experimentos en seres humanos ni en animales.

Confidencialidad de los datos. Los autores declaran que han seguido los protocolos de su centro de trabajo sobre la publicación de datos de pacientes.

Derecho a la privacidad y consentimiento informado. Los autores declaran que en este artículo si aparecen datos de pacientes.

\section{Bibliografía}

1. Jawetz E. Melnick JL, Adelberg EA. Medical mycology Actynomycetes. En: Review of medical microbiology. Lange Medical Publications; Los Altos, California, Estados Unidos, 1982. p. 301-2.

2. Bannura G. Actinomicosis abdominal. Rev Med Chil. 1994;122:1307-15.

3. Alegría J, González M, Galleguillos M, Whittle C, Franco C. Revisión de infección pélvica por actinomices: presentación de un caso clínico. Rev Chil Radiol. 2003;9:196-200.
4. Ovalle A, Casanova A, Kakarieka E, de Jourdan F, Salgado K. Epidemiología, resultados clínicos y costos del tratamiento del absceso tuboovárico, en un hospital público de Santiago. Rev Chil Obstet Ginecol. 2008;73:374-80.

5. Madrid F, Díaz S, Mucientes F, Klaassen R. Actinomicosis ginecológica. Rev Chil Obstet Ginecol. 2003;68:21-7.

6. Silva S. Procesos inflamatorios pelvianos. En: Infecciones en Ginecología y Obstetricia; Santiago Chile; Ed.Mediterráneo, 2010. p. 90-4.

7. Daniels P, Correa E, Goity C. Actinomicosis pélvica: caso clínico. Rev Chil Obstet Ginecol. 2013;78:48-50.

8. Fiorino AS. Intrauterine contraceptive device-associated actinomycotic abscess and Actinomyces detection on cervical smear. Obstet Gynecol.1996;87:142-9.

9. Lippes J. Pelvic actinomycosis: a review and preliminary look at prevalence. Am J Obstet Gynecol. 1999;180:265-9.

10. Westhoff C. IUDS and colonization or infection with Actinomyces. Contraception. 2007;75:48-50.

11. Taga S. Diagnosis and therapy of pelvic actinomycosis. J Obstet Gynaecol Res. 2007;33:882-5.

12. Pakish JB, West $\mathrm{L}$. Actinomyces bacteremia in association with tubo-ovarian abscesses and hysteroscopic sterilization. Obstet Gynecol. 2014;124 (2 Pt 2 Suppl 1):451-3.

13. Faúndez J, Uribe S, Pizarro F. Actinomicosis pélvica. A propósito de un caso que simula un tumor de recto. Rev Chil Cir. 2019;71:557-61.

14. Elsayed S, George A, Zhang K. Intrauterine contraceptive device-associated pelvic actinomycosis caused by Actinomyces urogenitalis. Anaerobe. 2006;12:67-70.

15. Carrillo M, Váldez B, Vargas L, Álvarez L, Schorr M, Zlatev R, et al. In vitro Actinomyces israelii biofilm development on IUD copper surfaces. Contraception. 2010;81:261-4.

16. Demitry A, Rao S, Cawdell G. An unusual case of abdominal actinomycosis. J Obstet Gynaecol. 2009;29:70-1.

17. Pusiol T, Morichetti D, Pedrazzani C, Ricci F. Abdominal-pelvic actinomycosis mimicking malignant neoplasm. Infect Dis Obstet Gynecol. 2011;2011:747-59

18. Bae JH, Song R, Lee A, Park JS, Kim MR. Computed tomography for the preoperative diagnosis of pelvic actinomycosis. J Obstet Gynaecol Res. 2011;37:300-4.

19. Bercovich A, Guy M, Karayiannakis AJ, Gdalia M, Muriel E, Dgani R, et al. Ureteral obstruction and reconstruction in pelvic actinomycosis. Urology. 2003;61:224.

20. Ugezu CH, Kelly I, Walker F, Stratton JF. A case of pelvic actinomycosis with bilateral hydronephrosis and renal failure associated with prolonged intrauterine contraceptive systems use. J Obstet Gynaecol. 2012;32:403-4.

21. Lawson E. Systemic actinomycosis mimicking pelvic malignancy with pulmonary metastases. Can Respir J. 2005;12:153-4.

22. O'Kelly K, Abu J, Hammond R, Jensen M, O'Connor RA, Soomro I. Pelvic actinomycosis with secondary liver abscess, an unusual presentation. Eur J Obstet Gynecol Reprod Biol. 2012;163:239-40.

23. Carrara J, Hervy B, Dabi Y, Illac C, Haddad B, Skalli D, et al. Added-value of endometrial biopsy in the diagnostic and therapeutic strategy for pelvic actinomycosis. J Clin Med. 2020;9:821.

24. González O, Motta G, Uscanga M, Aguilar R. Hallazgos tomográficos de la actinomicosis abdominopélvica. An Radiol Mex. 2007;3:179-88.

25. Heo SH, Shin SS, Kim JW, Lim HS, Seon HJ, Jung SI, et al. Imaging of actinomycosis in various organs: a comprehensive review. Radiographics. 2014;34:19-33.

26. Laios A, Terekh I, Majd HS, Pathiraja P, Manek S, Haldar K. Differentiating pelvic actinomycosis from advanced ovarian cancer: a report of two cases, management reflections and literature review. Gynecol Oncol Res Pract. 2014;1:5

27. Marret H, Wagner N, Ouldamer L, Jacquet A, Body G. [Pelvic actinomycosis: just think of it]. Gynecol Obstet Fertil. 2010;38:307-12.

28. Lerner PI. Susceptibility of pathogenic actinomycetes to antimicrobial compounds. Antimicrob Agents Chemother. 1974;5:302-9.

29. Smith AJ, Hall V, Thakker B, Gemmell CG. Antimicrobial susceptibility testing of Actinomyces species with 12 antimicrobial agents. J Antimicrob Chemother. 2005;56:407-9. 